KOBE-TH-93-10

November 1993

\title{
On the Poincaré Polynomials for Landau-Ginzburg Orbifolds
}

\author{
Hitoshi Sato \\ Graduate School of Science and Technology, Kobe University \\ Rokkodai, Nada, Kobe 65\%, Japan \\ email address : UTOSA@JPNYITP.BITNET
}

\begin{abstract}
We construct the Poincaré polynomials for Landau-Ginzburg orbifolds with projection operators. Using them we show that special types of dualities including Poincaré duality are realized under certain conditions. When Calabi-Yau interpretation exists, two simple formulae for Hodge numbers $h^{2,1}$ and $h^{1,1}$ are obtained.
\end{abstract}




\section{Introduction}

A compactified heterotic string theory with $N=1$ space-time supersymmetry requires an $N=2$ superconformal field theory (SCFT) for its internal space. Although at least $(2,0)$ world sheet supersymmetry is necessary to have $N=1$ space-time supersymmetry, models with $(2,2)$ supersymmetry are of special interest because they could have geometrical picture. In fact, a large class of such models with central charge $c=9$ allows a CalabiYau interpretation [1].

It has been observed that Landau-Ginzburg models can uncover the relation between $N=2$ SCFT and Calabi-Yau picture [2]. A Landau-Ginzburg model describes a chiral ring of $N=2$ SCFT. It is known that a large class of Landau-Ginzburg models with integral $U(1)$ charge states have Calabi-Yau interpretation by identifying a chiral primary state which has $U(1)$ charge $(\hat{c}-p, q)$ with a $(p, q)$ form on a Calabi-Yau manifold, where $\hat{c}=c / 3$. If Calabi-Yau interpretation exists, the number of non-singlet representations of $E_{6}$ can be obtained from the charge degeneracies of a Landau-Ginzburg model.

To get low generation models, Landau-Ginzburg (LG) orbifold models and corresponding Calabi-Yau orbifolds have recentry been considered. LG orbifolds were first considered by Vafa [4] and further results are obtained in ref. [5]. Under certain conditions LG orbifolds still have Calabi-Yau interpretation and many examples are found [6, 7, 8]. These

models reveal a special feature called mirror symmetry [9, 10, 11], which is expected to give

an effective method for calculating Yukawa couplings [12, 13]. same

In this paper we investigate the structures of LG orbifolds. To do this, Poincaré polynomials for LG orbifolds with projection operators are good tools. However, such Poincaré polynomials are not known except for the models divided by $U(1)$ projection operator $j$ only. So we first construct Poincaré polynomials with general projection operators for certain calsses of LG orbifolds. In geometrical picture there are some dualities of Hodge numbers on a Calabi-Yau manifold. In the case of LG orbifolds we can find, through calculations model by model, that there exist dualities which are equivalent to those of Calabi-Yau orbifolds. But it is not clear why they are realized. Using our Poincaré polynomial we show how special dualities of LG orbifolds are realized. Finally we obtain simple fomulae for dimensions of chiral prmary fields. These dimensions correspond to undetermined Hodge numbers $h^{1,1}$ and $h^{2,1}$, so they determine the generation number of a heterotic string model compactified on a Calabi-Yau manifold [14].

This paper is organized as follows; in section 2 we construct the Poincaré polynomials for LG orbifolds with projection operators. It is shown in section 3 that these polynomials satisfy special types of dualities. Two fomulae for dimensions of chiral primary fields are 
obtained in section 4 . We give conclusions and discussions in the last section.

\section{Construction of the Poincaré polynomial}

The Landau-Ginzburg description of chiral rings depends on the assumption that an $\mathrm{N}=2$ supersymmetric theory with a non-degenerate quasi-homogeneous superpotential $W\left(\lambda^{n_{i}} X_{i}\right)=\lambda^{d} W\left(X_{i}\right), i=1 \sim N$, flows to an $\mathrm{N}=2$ superconformal model at a renormalization group fixed point. The

charges of the chiral super fields $X_{i}$ are $q_{i}=n_{i} / d$ and the central charge is given by $c=3 \sum_{i}\left(1-2 q_{i}\right)$. The chiral ring $\mathcal{R}$ is a quotient ring defined by

$$
\mathcal{R}=\frac{\mathbb{C}\left[X_{i}\right]}{\left[\partial_{j} W\right]}
$$

and the Poincaré polynomial for $\mathcal{R}$ is given by [3]

$$
\operatorname{Tr}_{(c, c)}\left[t^{J_{0}} \bar{t}^{\bar{J}_{0}}\right]=\prod_{i} \frac{1-(t \bar{t})^{\left(1-q_{i}\right)}}{1-(t \bar{t})^{q_{i}}}
$$

The LG orbifolds are obtained by quotienting with an abelian symmetry group $G$ of $W\left(X_{i}\right)$, whose element $g$ acts as an $N \times N$ diagonal matrix;

$$
g: X_{i} \rightarrow e^{2 \pi i \theta_{i}{ }^{g}} X_{i} .
$$

Only the $X_{i}$ invariant under the above transformation, that is those for which $\tilde{\theta}_{i}^{g} \equiv$ $\theta_{i}{ }^{g}-\left[\theta_{i}{ }^{g}\right]$ vanishes contribute to the chiral ring in the $g$-twisted sector. For a geometrical interpretation the important thing is $U(1)$ projection operator $j$, which acts as $j: X_{i} \rightarrow$ $e^{2 \pi i q_{i}} X_{i}$.

Poincaré polynomials for such LG orbifolds have already been constructed by Intriligator and Vafa in ref. [4, [5]. But they do not contain informations about transformation proprties of the states under $g$, so that the states which shoud be projected out still remain. So it is necessary to construct the Poincaré polynomial for a LG orbifold with a projection operator in order to know the true chiral ring structure.

We will restrict ourselves to the following three types of polynomials or products of them for $W\left(X_{i}\right)$;

1. $X_{1}^{a_{1}}+\cdots+X_{n}^{a_{n}}$

2. $X_{1}^{a_{1}} X_{2}+X_{2}^{a_{2}} X_{3}+\cdots+X_{n}^{a_{n}} X_{1}$

3. $X_{1}^{a_{1}} X_{2}+X_{2}^{a_{2}} X_{3}+\cdots+X_{n}^{a_{n}}$. 
They are called Fermat, loop and tadpole type, respectively. These three types of $W\left(X_{i}\right)$ are very interesting because they contain A-D-E $N=2$ supersymmetric minimal models. Note that only these types appear in the Berglund-Hübsch construction of mirror manifolds 11.

In order to obtain Poincaré polynomial with $g$ we first construct the chiral ring (11) directly. Before explaining our method, let us briefly summarize the results in [4, 5]. The arbitrary (c,c) states in the $h$-twisted sector can be represented as

$$
\prod_{\tilde{\theta}_{i}^{h}=0}\left(X_{i}\right)^{\ell_{i}}|0\rangle_{(c, c)}^{(h)}
$$

with appropriate $\ell_{i}$. The transformation properties of chiral primary states have been investigated from modular invariance of the Witten index and they are given by

$$
g \prod_{\tilde{\theta}_{i}{ }^{h}=0}\left(X_{i}\right)^{\ell_{i}}|0\rangle_{(c, c)}^{(h)}=e^{2 \pi i \phi^{g}(h)}\left(\exp 2 \pi i \sum_{\tilde{\theta}_{i}{ }^{h}=0} \tilde{\theta}_{i}^{g} \ell_{i}\right) \prod_{\tilde{\theta}_{i}{ }^{h}=0}\left(X_{i}\right)^{\ell_{i}}|0\rangle_{(c, c)}^{(h)}
$$

where

$$
e^{2 \pi i \phi^{g}(h)}=\left.\epsilon(g, h)(-1)^{K_{g} K_{h}}(\operatorname{det} g)^{-1} \operatorname{det} g\right|_{h}
$$

and

$$
\left.\operatorname{det} g\right|_{h}=\exp \left(2 \pi i \sum_{\tilde{\theta}_{i}^{h}=0} \tilde{\theta}_{i}^{g}\right) .
$$

$\epsilon(g, h)$ is the discrete torsion and $K_{g}$ is an integer defined mod 2. They are not determined by modular invariance of the Witten index. But they must satisfy certain conditions to get integral left and right charges. We will demand such conditions in later sections.

The charges of the state $|0\rangle_{(c, c)}^{(h)}$ were obtained [4] to be

$$
\left(\begin{array}{c}
J_{0} \\
\bar{J}_{0}
\end{array}\right)|0\rangle_{(c, c)}^{(h)}=\left(\sum_{\tilde{\theta}_{i}{ }^{h}>0}\left(\frac{1}{2}-q_{i}\right) \pm\left(\tilde{\theta}_{i}{ }^{h}-\frac{1}{2}\right)\right)|0\rangle_{(c, c)}^{(h)} .
$$

Now we turn our attention to the Poincaré polynomial. To illustrate our construction we consider a Fermat type as an example. For a Fermat type polynomial, the chiral ring $\mathcal{R}$ is determined by the equations

$$
X_{i}^{a_{i}-1}=0 .
$$


and chiral primary states are in the form $\prod_{i} X_{i}^{\ell_{i}}$ with $0 \leq \ell_{i} \leq a_{i}-2$. To construct a Poincaré polynomial it is useful to introduce the notations $x_{i} \equiv t^{q_{i}}$. In this case it is easy to see that the Poincaré polynomial is given by

$$
P(t)=\frac{x_{1}^{a_{1}-1}-1}{x_{1}-1} \frac{x_{2}^{a_{2}-1}-1}{x_{2}-1} \cdots \frac{x_{n}^{a_{n}-1}-1}{x_{n}-1} .
$$

Twisted sectors appear when the Landau-Ginzburg theory is orbifoldized. In the $h$ twisted sector we must consider the following restricted polynomial

$$
\prod_{\tilde{\theta}_{i}^{h}=0} \frac{x_{i}^{a_{i}-1}-1}{x_{i}-1}
$$

When $g$ acts on this chiral ring, the Poincaré polynomial gets phases according to eq.(5). These phases are obtained by transformations

$$
x_{i} \rightarrow e^{2 \pi i \tilde{\theta}_{i}{ }^{g}} x_{i}
$$

except for the overall phase $e^{2 \pi i \phi^{g}(h)}$, which is defined below (5).

Then we obtain the Poincaré polynomial for $(c, c)$ states of the $h$-twisted sector,

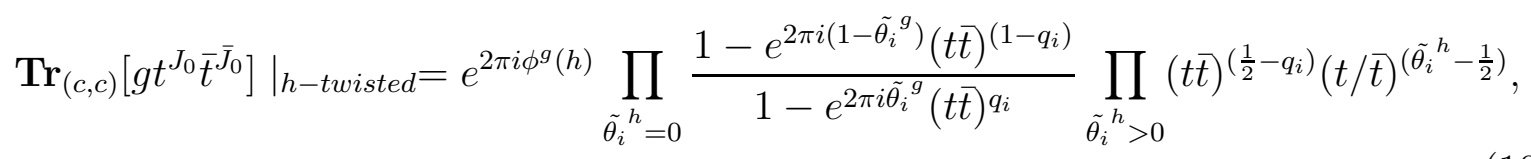

where we have changed the variables from $x_{i}$ to $t^{q_{i}}$ and the trace has been taken over the $h$-twisted sector only. The last product arises due to (6). For loop and tadpole type polynomials we can get the same results [15].

Consequently we can construct the Poincaré polynomial with the projection operator $\hat{P} \equiv \frac{1}{|G|} \sum($ all $g \in G)$

$$
P(t, \bar{t}) \equiv \operatorname{Tr}_{(c, c)}\left[\hat{P} t^{J_{0}} \bar{t}^{\bar{J}_{0}}\right],
$$

where we have taken the trace over all the sectors. This Poincaré polynomial contains informations on projected (c,c) states only. The coefficients of the Poincaré polynomial $p_{i, j}$ defined by $P(t, \bar{t})=\sum p_{i, j} t^{i} \bar{t}^{j}$ are the dimensions of the chiral primary fields with charge $(i, j)$.

\section{Special dualities}

It is well known that Hodge numbers of a Calabi-Yau 3-fold obey the following relations, which we call special dualities [20]; 
1. Poincaré duality; $h^{i, j}=h^{3-i, 3-j}$

2. Complex conjugation duality; $h^{i, j}=h^{j, i}$

3. Holomorphic duality; $h^{0, j}=h^{0,3-j}, h^{i, 0}=h^{3-i, 0}$.

We will show that for $p_{i, j}$ the same special dualities hold if an LG orbifold has a geometrical interpretation. Due to the identifications $p_{\hat{c}-i, j}=h^{i, j}$, these dualities are 1 . $p_{i, j}=p_{\hat{c}-i, \hat{c}-j} ; 2 . p_{\hat{c}-i, j}=p_{\hat{c}-j, i}$ and 3. $p_{\hat{c}, j}=p_{\hat{c}, 3-j}, p_{\hat{c}-i, 0}=p_{i, 0}$. In the following we set $c=9$. To see these dualities we should know which twisted sector contributes to $p_{i, j}$. We first consider $p_{i, 0}$ and $p_{0, i}(i=1,2)$, because some LG orbifolds could have non-vanishing ones.

From our Poincaré polynomial (10) nonzero $p_{2,0}$ can arise only from the twisted sector with both $\sum_{\tilde{\theta}_{i}{ }^{h}>0}\left(\tilde{\theta}_{i}{ }^{h}-q_{i}\right)=2$ and $\sum_{\tilde{\theta}_{i}{ }^{h}>0}\left(1-\tilde{\theta}_{i}{ }^{h}-q_{i}\right)=0$. Using the similar discussion by Kreuzer and Skarke [6], we can show that $\sum_{\tilde{\theta}_{i}{ }^{h}>0}\left(1-\tilde{\theta}_{i}{ }^{h}-q_{i}\right)=0$ implies $\left(1-\tilde{\theta}_{i}{ }^{h}-\right.$ $\left.q_{i}\right)=0$

for $\tilde{\theta}_{i}{ }^{h}>0$ 15, so that in this case we have $\sum_{\tilde{\theta}_{i}{ }^{h}>0}\left(1-2 q_{i}\right)=2$. We therefore conclude that nonzero $p_{2,0}$ can exist only if the following two conditions are satisfied;

1. There is at least one element $j_{2}$ of the symmetry group $G$ such that $j_{2}$ acts like $j$ on the fields $X_{i} \in \mathcal{M}_{2}$ (where $\mathcal{M}_{2}$ is a subset of the fields $X_{i}$ ) and does not act at all on the other fields.

2. The contribution of the fields in $\mathcal{M}_{2}$ to the central charge is 6 , i.e. $\sum_{X_{i} \in \mathcal{M}_{2}}\left(1-2 q_{i}\right)=$ 2.

Thus only the $j_{2}^{-1}$-twisted sector can contribute to $p_{2,0}$ and, by the above definition, only the $j^{-1} j_{2}$-twisted sector can contribute to $p_{1,0}$.

We can see that the operator $j j_{2}^{-1}$ is nothing but the $j_{1}$ in ref. [7]. It was shown that nonzero $p_{0,1}$ and $p_{0,2}$ can exist only if there is at least one element $j_{1}$ of $G$ [7]. So we see that $p_{1,0}, p_{2,0}, p_{0,1}$ and $p_{0,2}$ can be nonzero only if the element $j_{1}$ of $G$ exists.

The above discussion allows us to find the twisted sectors which contribute

to other $p_{i, j}$. In the $h$-twisted sector we can see through the Poincaré polynomial (10) that there

is one state with the lowest charges $\left(\sum_{\tilde{\theta}_{i}{ }^{h}>0}\left(\tilde{\theta}_{i}{ }^{h}-q_{i}\right), \sum_{\tilde{\theta}_{i}{ }^{h}>0}\left(1-\tilde{\theta}_{i}{ }^{h}-q_{i}\right)\right)$ and that only one state with the highest charges $\left(Q+\sum_{\tilde{\theta}_{i}{ }^{h}>0}\left(\tilde{\theta}_{i}{ }^{h}-q_{i}\right), Q+\sum_{\tilde{\theta}_{i}{ }^{h}>0}\left(1-\tilde{\theta}_{i}{ }^{h}-q_{i}\right)\right)$ exists, where $Q=\sum_{\tilde{\theta}_{i}{ }^{h}=0}\left(1-2 q_{i}\right)$. Then we obtain the results listed in Table 1 .

It was shown in [5] that, to get an LG orbifold with integral left and right charges, we must take $G$ containing $j$ and require $\epsilon(j, g)=(-1)^{K_{j} K_{g}} \operatorname{det} g$ and $(-1)^{K_{g}}=\operatorname{det} g$. For 


\begin{tabular}{||c|c||}
\hline$p_{i, j}$ & twisted sector \\
\hline \hline$p_{0,0}, p_{3,3}$ & untwisted \\
\hline$p_{0,3}$ & $j$ \\
\hline$p_{3,0}$ & $j^{-1}$ \\
\hline$p_{0,1}, p_{2,3}$ & $j_{1}$ \\
\hline$p_{1,0}, p_{3,2}$ & $j_{1}^{-1}$ \\
\hline$p_{0,2}, p_{1,3}$ & $j j_{1}^{-1}$ \\
\hline$p_{2,0}, p_{3,1}$ & $\left(j j_{1}^{-1}\right)^{-1}$ \\
\hline$p_{2,1}$ & $h_{1} \cdots$ \\
\hline$p_{1,2}$ & $h_{1}^{-1} \cdots$ \\
\hline$p_{1,1}, p_{2,2}$ & $h_{2} \cdots$ \\
\hline
\end{tabular}

Table 1: The twisted sectors contributing to $p_{i, j} . h_{1}, \cdots\left(h_{2}, \cdots\right)$ indicate the twisted sectors that contribute to $p_{2,1}\left(p_{1,1}\right.$ and $\left.p_{2,2}\right)$.

geometrical interpretation we further require $\operatorname{det} g=1, \epsilon(g, h)=(-1)^{K_{g}}=1$ for all $g$ and $h$ in $G$. Hence we have $\operatorname{det} j=1$ and this implies the number of

fields $N=$ odd for $c=9$. The requirement $\operatorname{det} g=1$ for all $g \in G$ is needed to guarantee the invariance of holomorphic three form [12]. In the remaining part of this section we assume these requirements to hold.

We can see through the Poincaré polynomial (10) that in the $h$-twisted sector the phase of the state with the lowest charges is $\left.\operatorname{det} g\right|_{h}$ and the one with the highest charges is $\left(\left.\operatorname{det} g\right|_{h}\right)^{-1}$. Then we find that the $(\mathrm{c}, \mathrm{c})$ states have the phases listed in Table 2, if they exist.

By definition $\left.\operatorname{det} g\right|_{j_{1}}=\left.\operatorname{det} g\right|_{j_{1}-1}$, and owing to $\operatorname{det} g=1$ for all $g \in G$ we obtain $\left.\operatorname{det} g\right|_{j_{1}}=\left(\left.\operatorname{det} g\right|_{j j_{1}-1}\right)^{-1}$. Therefore we see that if one of the above states survives after projection then all the other states must do. So the set of $p_{i, j}$ with the above charges satisfies special dualities. It can be shown through the observation of the phases in eq.(10) that if $(2,1)$ states in the $h_{1}$-twisted sector survive then corresponding $(1,2)$ states in the $h_{1}{ }^{-1}$-twisted sector must survive, or vice versa. We can see that there are the same structures between $(1,1)$ and

$(2,2)$ states. Therefore each set of $\left\{p_{2,1}, p_{1,2}\right\}$ and $\left\{p_{1,1}, p_{2,1}\right\}$ respects the dualites 1. and 2. Consequently we have special dualities for the LG orbifold corresponding to Poincaré duality, complex conjugation duality and holomorphic duality. 


\begin{tabular}{|c|c|}
\hline state & phase \\
\hline$(0,1)$ & $\left.\operatorname{det} g\right|_{j_{1}}=\prod_{\tilde{\theta}_{i}{ }^{j_{1}}=0} e^{2 \pi i \tilde{\theta}_{i}^{h}}$ \\
\hline$(2,3)$ & $\left(\left.\operatorname{det} g\right|_{j_{1}}\right)^{-1}=\prod_{\tilde{\theta}_{i}{ }^{j_{1}}=0} e^{-2 \pi i \tilde{\theta}_{i}^{h}}$ \\
\hline$(0,2)$ & $\left.\operatorname{det} g\right|_{j j_{1}-1}=\prod_{\tilde{\theta}_{i}^{j j_{1}-1}=0} e^{2 \pi i \tilde{\theta}_{i}^{h}}$ \\
\hline$(1,3)$ & $\left(\left.\operatorname{det} g\right|_{j j_{1}-1}\right)^{-1}=\prod_{\tilde{\theta}_{i}^{j j_{1}-1}=0} e^{-2 \pi i \tilde{\theta}_{i}^{h}}$ \\
\hline$(2,0)$ & $\left.\operatorname{det} g\right|_{\left(j j_{1}-1\right)^{-1}}=\prod_{\left.\tilde{\theta}_{i}^{\left(j j_{1}-1\right.}\right)^{-1}=0} e^{2 \pi i \tilde{\theta}_{i}^{h}}$ \\
\hline$(3,1)$ & $\left(\left.\operatorname{det} g\right|_{\left(j j_{1}-1\right)^{-1}}\right)^{-1}=\prod_{\left.\tilde{\theta}_{i}^{\left(j j_{1}\right.}-1\right)^{-1}=0} e^{-2 \pi i \tilde{\theta}_{i}^{h}}$ \\
\hline$(1,0)$ & $\left.\operatorname{det} g\right|_{j_{1}-1}=\prod_{\tilde{\theta}_{i}^{j_{1}^{-1}=0}} e^{2 \pi i \tilde{\theta}_{i}^{h}}$ \\
\hline$(3,2)$ & $\left(\left.\operatorname{det} g\right|_{j_{1}-1}\right)^{-1}=\prod_{\tilde{\theta}_{j}^{j_{1}-1}=0} e^{-2 \pi i \tilde{\theta}_{i}^{h}}$ \\
\hline
\end{tabular}

Table 2: The phases of the states with charges $(i, j)$.

\section{Formulae for $p_{1,1}$ and $p_{2,1}$}

In this section we consider the $p_{1,1}$ and $p_{2,1}$ through the Poincaré polynomial (11). Let $n_{27}$ and $n_{27}$ be the number of $\mathbf{2 7}$ and $\overline{\mathbf{2 7}}$ representations of $E_{6}$ in a heterotic string model. If we have a Calabi-Yau interpretation, then $p_{1,1}=n_{27}$ and $p_{2,1}=n_{2 \overline{7}}$. So they determine the generation number to be $\left|p_{2,1}-p_{1,1}\right|[14]$.

For an LG orbifold with integral left and right charges we have using the Poincaré polynomial (11),

$$
P(-1,-1)=\frac{1}{|G|} \sum_{i}(-1)^{N+K_{g} K_{h}+K_{g h}} \epsilon(g, h) \prod_{\tilde{\theta}_{i}^{g}=\tilde{\theta}_{i}^{h}=0}\left(1-\frac{1}{q_{i}}\right),
$$

where $K_{g h}=K_{g}+K_{h}$. In the following we consider LG orbifolds with Calabi-Yau interpretations. So we put the same conditions in section 3 and we have $P(-1,-1)=$ $2\left(p_{1,1}-p_{2,1}\right)$. The identifications $p_{1,1}=h^{2,1}$ and $p_{2,1}=h^{1,1}$ imply $P(-1,-1)=-\chi$.

In ref. [7] it was shown that the exponent of $t$ in a Poincaré polynomial is non-negative,

$$
\sum_{\tilde{\theta}_{i}^{h}>0}\left(\tilde{\theta}_{i}^{h}-q_{i}\right) \geq 0
$$

By a similar discussion we can also show that the exponent of $\bar{t}$ is non-negative [15],

$$
\sum_{\tilde{\theta}_{i}^{h}>0}\left(1-\tilde{\theta}_{i}^{h}-q_{i}\right) \geq 0
$$


From these inequalities we obtain

$$
-\frac{\hat{c}}{2} \leq \sum_{\tilde{\theta}_{i}^{h}>0}\left(\tilde{\theta}_{i}^{h}-\frac{1}{2}\right) \leq \frac{\hat{c}}{2} .
$$

Let us denote by $I$ the number of invariant fields in the $h$-twisted sector. If $I=$ odd, $\sum_{\tilde{\theta}_{i}{ }^{h}>0}\left(\tilde{\theta}_{i}^{h}-\frac{1}{2}\right)=0, \pm 1$.

We consider LG orbifolds with $\sum_{\tilde{\theta}_{i}>0}\left(\tilde{\theta}_{i}-\frac{1}{2}\right) \neq \pm 1$ in all the twisted sectors (namely LG orbifolds without $j_{1}$ ) since the generation number of the LG orbifolds with nonzero $p_{0,1}$ vanishes [6, 7]. We see that chiral primary states in the sector with $I=$ odd contribute to $p_{i, i}$ for $i=0 \sim 3$ and in the sector with $I=$ even contribute to other $p_{i, j}$. Using the above discussions and structures of $p_{i, j}$, we have

$$
\begin{aligned}
& p_{1,1}=\left\{\frac{-1}{2} \frac{1}{|G|} \sum_{\text {allg } \in G} \sum_{\substack{h \in G \\
I=\text { odd }}} \prod_{i}^{g} \prod_{i}\left(1-\frac{1}{q_{i}^{h}}\right)\right\}-1 \\
& p_{2,1}=\left\{\frac{1}{2} \frac{1}{|G|} \sum_{\text {allg } \in G} \sum_{\substack{h \in G \\
I=\text { even }}} \prod_{\tilde{\theta}_{i}^{g}=\tilde{\theta}_{i}^{h}=0}\left(1-\frac{1}{q_{i}}\right)\right\}-1 .
\end{aligned}
$$

(If the set of $i$ 's satisfying $\tilde{\theta}_{i}^{g}=\tilde{\theta}_{i}{ }^{h}=0$ is empty, we define $\prod_{\tilde{\theta}_{i}^{g}=\tilde{\theta}_{i}^{h}=0}\left(1-\frac{1}{q_{i}}\right)=1$.)

It is a remarkable fact that we can calculate these numbers without any information on actual structure of the chiral ring. These results are natural extentions of the formulae obtained by Roan [16]. He got simple formulae for Betti numbers of resolved Calabi-Yau manifolds with $d=\sum n_{i}$ in $W C P^{4}$, i.e. in the case of $N=5$ with $\sum q_{i}=1$. Our formulae

are applicable to other types of resolved Calabi-Yau manifolds, say Schimmrigk's construction of the Tian-Yau manifold and its orbifolds

[18, 19] which can be described by $W\left(X_{i}\right)$ with $N=7$ and $\sum q_{i}=2$.

We can obtain the above results by the method of ref. [7], where $P(1,1)$ was used for calculation as well as $P(-1,-1)$. It is easy to compute $P(1,1)$ from our Poincaré polynomial with projection operator and we get the same result as in ref. [7].

\section{Conclusions and discussions}

In this paper we have constructed the Poincaré polynomials with projection operators for general abelian LG orbifolds. Although we considered only three types of potentials, we believe that the same results hold for general $W\left(X_{i}\right)$ [15]. If Calabi-Yau interpretation exists, special dualities of $p_{i, j}$ are realized and simple formulae for $p_{1,1}$ and $p_{2,1}$ are obtained. 
It is worth pointing out that our results will be useful to examie Yukawa couplings. The geometrical method for Yukawa couplings are considered in [12, 13] and it is a challenging problem to calculate them in terms of LG orbifold technique. Recent attempts are found in [17]. To evaluate $27^{3}$ Yukawa couplings, we must first get informations about $(1,1)$ states. Using our formula for $p_{1,1}$ we can see which twisted sector coud have $(1,1)$ states, without concrete representations of $(1,1)$

states. Then we can apply the twist number selection rule to $\mathbf{2 7 ^ { \mathbf { 3 } }}$ Yukawa couplings and get non-vanishing ones.

Acknowledgements : The auther would like to thank Professor M. Ida for helpful discussions and for careful reading of this manuscript.

\section{References}

[1] D.Gepner,Phys.Lett.199B(1987)380

[2] B.R. Greene, C. Vafa and N.P. Warner,Nucl. Phys.B324(1989)371

[3] W. Lerche, C. Vafa and N.P. Warner,Nucl Phys.B324(1989)427

[4] C.Vafa,Mod.Phys.Lett.A4(1989)1169

[5] K. Intriligator and C. Vafa,Nucl. Phys.B339(1990)95

[6] M. Kreuzer and H. Skarke,Nucl. Phys.B388(1992)113

[7] M.Kreuzer and H.Skarke,Nucl. Phys. B405(1993)305

[8] M.Krezer, R. Schimmrigk and H.Skarke,Nucl.Phys.B372(1992)61

[9] P. Candelas, M. Lynker and R.Schimmrigk,Nucl. Phys.B341(1990)383

[10] B.R. Greene and M.R. Plesser,Nucl Phys.B338(1990)15

[11] P. Berglund and T. Hübsch,Nucl. Phys.B393(1993)377

[12] P.Candelas, Nucl. Phys.B298(1988)458.

[13] P. Candelas, X. de la Ossa, P.S. Green and L. Parkes, Phys. Lett 258B(1991)118 and Nucl. Phys. B359(1991)21

[14] P.Candelas, G.Horowitz, A.Strominger and E.Witten,Nucl Phys. B258(1985)46.

[15] H. Sato, in preparation. 
[16] S.S. Roan,Internat.J.Math.(4)2(1991)439

[17] P.Berglund and T.Hübsch,HUPAPP-93/2,NSF-ITP-93-37,UTTG-10-93.

[18] R. Schimmrigk, Phys. Lett.193B(1987)175

[19] P.S. Aspinwall and C.A. Lütken, Nucl. Phys.B353(1991)427

[20] T.Hübsch: Calabi-Yau manifolds - A Bestiary for Physicists (World Scientific, Singapore, 1992). 